\title{
A FAMILY OF THE SPIRAL SOLUTIONS OF THE NONLINEAR KLEIN-GORDON EQUATION
}

\author{
V. V. GUDKOV \\ Institute of Mathematics and Computer Science, \\ University of Latvia, \\ Riga LV-1459, Latvia
}

\begin{abstract}
A family of the functions, intended for a construction the exact travelling wave solutions of nonlinear partial differential equations, is given. Exact solutions of the Klein-Gordon equation with a special potential are obtained. The behavior of complex and hypercomplex solutions of the second order is presented.
\end{abstract}

\section{INTRODUCTION}

In this paper a short analysis of the family $\{q\}$ of the functions, intended for a construction the exact travelling wave solutions of partial differential equations (PDEs), is given. The first function (inflection function), which is denoted $q_{0}$, was defined in [1]. Due to this function the exact travelling wave solutions of some reaction-diffusion equations were obtained. The second function (complex function), which is denoted $q_{1}$, was defined in [2]. The hypercomplex functions $q_{n}$, defined later [3], filled out the family $q$. The corresponding family $2 q-1$ of the exact travelling wave solutions of the KleinGordon (KG) equation was obtained in [4]. The relations between hypercomplex solutions and Special Unitary Groups $S U(n)$ was given in [5]. Due to the family $\{q\}$ the exact solutions for a lot of the nonlinear PDEs, encountered in different areas of physics, were btained.

On a base of the family $\{2 q-1\}$ in this paper we construct the exact solutions of the KG equation with more general potential. The behavior of two solutions (complex and hypercomplex of the second order) of this equation is given. It is important to stress that the KG equation describes a two level system, and hence the given below solutions characterize the transfer from one level to another one. 
The paper is organized as follows. In Sec. 2 we write out the explicit expressions of the functions $q$. In Sec. 3 we describe the KG equation with special potential and give a family of its exact solutions. In Sec. 4 we show the behavior of the complex and hypercomplex of the second order solutions. And finally, in Sec. 5 some applications are given.

\section{THE FAMILY $\{Q\}$}

We deal with a class of integrable PDEs. There are several methods for obtaining the exact solutions of those equations. For the purposes of the method of substitutions, we have proposed a family $\{q\}$ of the functions, defined and constructed in our previous papers. An application of the polynomials on these functions makes this method more efficient. Due to the fact, that the family contains not only real functions but also complex and hypercomplex functions, we have extended a class of exact solutions for a large class of the integrable equations.

Let us write out the functions $q$ of real variable $z$. Two real functions are defined as

$$
q_{0}(z)=\frac{1}{1+\exp (z)}, \quad q_{*}(z)=\frac{1}{1-\exp (z)} .
$$

Two complex conjugate functions are defined as

$$
q_{1}(z)=q_{0}(2 z) \pm i \frac{\operatorname{sech}(z)}{2} .
$$

The hypercomplex functions of the second order are defined as

$$
q_{2}(z)=q_{0}(2 z) E_{2} \pm \frac{\operatorname{sech}(z)}{2} \sum_{j=1}^{3} a_{j} M_{j}, \quad \sum_{j=1}^{3} a_{j}^{2}=1,
$$

where the hypercomplex numbers $M_{j}$ are the quaternions [6]

$$
\begin{gathered}
E_{2}=\left(\begin{array}{ll}
1 & 0 \\
0 & 1
\end{array}\right), M_{1}=\left(\begin{array}{cc}
0 & i \\
i & 0
\end{array}\right), \\
M_{2}=\left(\begin{array}{cc}
0 & -1 \\
1 & 0
\end{array}\right), M_{3}=\left(\begin{array}{cc}
i & 0 \\
0 & -i
\end{array}\right) .
\end{gathered}
$$

The hypercomplex functions of $n$-th order are defined as

$$
q_{n}(z)=q_{0}(2 z) E_{n} \pm \frac{\operatorname{sech}(z)}{2} \sum_{j=1}^{m} a_{j} M_{j}, \quad \sum_{j=1}^{m} a_{j}^{2}=1 .
$$


Here $E_{n}, M_{1}, \ldots, M_{m}$ is a set of hypercomplex numbers of $n$-th order, $E_{n}$ is a unit and the numbers $M_{j}$ possess the following properties:

$$
\begin{gathered}
M_{i} M_{j}=-M_{j} M_{i} \text { for } i \neq j, \\
M_{j} M_{j}=-E_{n} \text { for } i, j=1,2, \ldots, m .
\end{gathered}
$$

Note that, for the matrix representation of the numbers $M_{j}$, the index $n$ corresponds to an order of $n \times n$-matrix $M_{j}$. Also, the index $m$ is equal to a number of linear-independent matrices $M_{j}$ that are unitary $\left(M_{j}^{-1}=\right.$ $\left.M_{j}^{*}\right)$, anti-Hermitian $\left(M_{j}^{*}=-M_{j}\right)$, and anticommuting $\left(M_{i} M_{j}=-M_{j} M_{i}\right)$ matrices. A symbol $*$ denotes a passage to the complex conjugate trasposed matrix. It is known that $m=3$ for $n=2, m=1$ for $n=3$, and $m=4$ for $n=4$.

A fundamental property of the functions $q$ is that any function $q$ on the family $\{q\}$ satisfies the logistic equation $q^{\prime}=q^{2}-q$ (Theorem 1 in [4]). It follows, that $n$-th derivative on $q^{k}$ is expressed by polynomial of $(n+k)$-th order on $q$. A basic property of the functions $q$ formulates as follows: if the polynomial function

$$
u=c_{0} q^{n}+c_{1} q^{n-1}+c_{2} q^{n-2}+\cdots+c_{n}
$$

satisfies a PDE for $q=q_{0}$, then $u$ satisfies the equation for any $q$ from the family $\{q\}$.

\section{KLEIN-GORDON EQUATION}

Now we investigate the KG equation in one space dimension with special potential

$$
u_{x x}-u_{t t}=Q^{\prime}(u), \quad Q(u)=\frac{\lambda}{4}\left(u^{2}\right)^{\frac{k-1}{k}}\left(\left(u^{2}\right)^{\frac{1}{k}}-1\right)^{2},
$$

for $k=1,2, \ldots ; \quad \lambda>0$. For moving frame of reference $z=x-v t$, where $v$ is a velocity, we reduce the equation (1) to the ordinary differential equation

$$
\left(1-v^{2}\right) u^{\prime \prime}=\frac{\lambda}{2}\left(\frac{k+1}{k} u^{\frac{k+2}{k}}-2 u+\frac{k-1}{k} u^{\frac{k-2}{k}}\right) .
$$

On the base of the family $\{q\}$ we construct the exact solutions of the equation (2) and hense of the equation (1). They are

$$
u(z ; n, k)=\left(2 q_{n}( \pm \alpha z / k)-E_{n}\right)^{k}, \quad n, k=1,2, \ldots,
$$

where

$$
\alpha=\sqrt{2 \lambda /\left(1-v^{2}\right)}, \quad v^{2}<1 .
$$


This result can be verified by direct substitution of functions (3) to the equation (2).

This family includes the family $\{2 q-1\}$ of the exact travelling wave solutions for the KG equation with potential $U=(\lambda / 4)\left(u^{2}-1\right)^{2}$ (particular case of $Q$ for $k=1$ ). A real solution of the KG equation with this particular choise of the potential have been known for a long time $[7 ; 8]$, this is so-called kink-solution $u_{0}=\tanh (-\alpha z / 2)$. It follows from the property $2 q_{0}(\alpha z)-1=\tanh (-\alpha z / 2)$, that the kink-solution belongs to the family (3).

The KG equation with potential $U$ is usually considered together with the boundary conditions of two level system, i.e. $u=-1$ for $z \rightarrow-\infty$ and $u=1$ for $z \rightarrow \infty$. The KG equation with a special potential, for example for even $k$, can be considered in addition with equal boundary values.

\section{THE BEHAVIOR OF THE SOLUTIONS}

In this section we show the behavior of the complex and hypercomplex of the second order solutions. Let

$$
\phi(\alpha z)=\operatorname{arccot}(\sinh (-\alpha z / k)) .
$$

Then from (3) for $n=1$ we take the solution

$$
u_{1}=\left(2 q_{1}(\alpha z / k)-1\right)^{k}=\exp (i k \phi) .
$$

Let $\left(a_{1}, a_{2}, a_{3}\right)=(0,0,1)$, then from (3) for $n=2$ we take the solution

$$
u_{2}=\operatorname{diag}(\exp (-i k \phi), \exp (i k \phi)) .
$$

In our previous papers $[4 ; 5]$ it is proved that the matrix solution $u(z ; 2,1)$ is an element of the Special Unitary Group $S U(2)$ of spinorial rotations. It is easy to verify that the matrix solution $u(z ; 2, k)$ is an element of $S U(2)$ also. It follows that the matrix $u(z ; 2, k)$ rotates the unit sphere around the vector $\left(a_{1}, a_{2}, a_{3}\right)$, and the matrix-solution $u_{2}$ rotates the unit sphere around the vector $(0,0,1)$. Note, that if the phase of the solution $u_{1}$ changes on $\theta$, then the unit sphere turns on angle $2 \theta$.

The solution $u_{1}$ is represented as a spiral curve in the system of coordinates $(\Re u, \Im u, z)$. For even integer $k$ of coordinates $(\Re u, \Im u, z)$ this curve has $k / 2$ loops. If the center of the unit sphere moves on this curve, then the rotational sphere derive a tube around the wave $u_{1}$. During this process any separate point on the sphere forms a spiral around the wave $u_{1}$. As the result we have a total set of the spiral curves, winding around wave $u_{1}$, i.e. we have a full tube, consisting of these spirals. Thus we can say, that the solution $u_{2}$ forms a surrounding field around the wave $u_{1}$. 


\section{SOME APPLICATIONS}

Davydov $[8 ; 9]$ has described a problem of electron transfer across biomembrane along $\alpha$-spiral protein molecule, namely along a chain of the peptide groups (PGs), which are connected by the hydrogen bonds. In the simple case a potential field among two adjacent PGs can be described by potential $U$, mentioned above. In the general case, when a deviation of PGs was taken in consideration, Davydov [9] has described a model of electron transfer by system of two equations. As a result, he describes an electron transfer by the wave function (see $[9, \mathrm{p} .99]$ )

$$
\psi(x, t)=\Phi(z) \exp (i(k x-\omega t)), \quad \Phi=A \operatorname{sech}(\alpha z) .
$$

Our approach allows us to consider the Davydov's solution together with a matrix solution. Hense we have got a surrounding field around the wave $\exp (i(k x-\omega t))$ inside the region $(-\Phi, \Phi)$. Such enclosing is a significant addition to Davydov's electrosoliton solution. A validity of this matrix solution is based on the following evident lemma.

Lemma 5.1. If the plane wave $A \exp ( \pm i(k x-\omega t))$ satisfies the linear differential equation, then the matrix function

$$
u(x, t)=A \operatorname{diag}(\exp (-i(k x-\omega t)), \exp (i(k x-\omega t)))
$$

also satisfies this equation.

It follows from this lemma that a plane wave exists together with its surrounding field, which is formed from the infinity set of spirals, twisted around plane wave.

In the case, when we describe the model of electron transfer along $\alpha$-spiral protein molecule, using KG equation with special potential, we construct a surrounding field around spiral wave $u_{1}$. Moreover using phase lag we can construct the three twisted one around another surrounding fields, corresponding to possible pathes of an external electron along the three chains of hydrogen bonds.

Another possible application of the family of exact solutions (3) can take place in physics of the elementary particles. Here we started from an observation, that the spiral $u_{1}$ characterizes a trajectory of moving point from one level (state) to another. The hypercomplex solution $u_{2}$ forms a surrounding field, which consists on a family of the spirals. A combination of these spirals can be useful for the electroweak currents description in the reactions of the particles decay.

\section{Acknowledgment}

This research was supported by the Latvian Council of Science, Grant No. 96. 0145 . 


\section{REFERENCES}

[1] V.V. Gudkov. Travelling-wave solutions for two-component reactive - diffusive systems. Comp. Maths Math. Phys. 35, No.4 (1995), P. 487-493.

[2] V.V. Gudkov. The explicit form of wave solutions of the evolutionary equations. Comp. Maths Math. Phys. 1996. 36, No. 3 (1996), P. 335-340.

[3] V.V. Gudkov. New types of wave solutions to the general nonlinear Klein equation. Comp. Maths Math. Phys. 37, No. 5 (1997), P. 584-589.

[4] V.V. Gudkov. A family of exact travelling wave solutions to nonlinear evolution and wave equations. J. Math. Phys. 38 No. 9 (1997), P. 4794-4803.

[5] V.V. Gudkov. On the relation between hypercomplex solutions and special unitary groups. Theor. Math. Phys. 113, No. 1 (1997), P. 1231-1234.

[6] E. Madelung Die Mathematischen Hilfsmittel des Physikers. Springer-Verlag, Berlin 1957.

[7] R.K. Dodd, J.C. Eilbek, J.D. Gibbon, H.C. Morris. Solitons and nonlinear wave equations. Mir, Moscow (1988). (in Russian)

[8] A.S. Davydov. The solitons in bioenergetics. Naukova dumka, Kiev (1988). (in Russian)

[9] A.S. Davydov Solitons in molecular systems. 1988. Naukova dumka, Kiev. (in Russian) 